\title{
GALACTIC BA ENRICHMENT FROM TP-AGB STARS
}

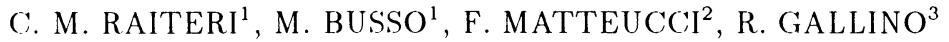 \\ 1 Osservatorio Astronomico di Torino, 10025 Pino Torinese, Italy \\ 2 ESO, Karl-Schwarzschild-Str.2, D-8046 Garching bei München, Germany
}

and

${ }^{3}$ Istituto di Fisica Generale, Università di Torino, Via P.Giuria 1, 10125 Torino, Italy

The production of the bulk of barium has long been ascribed to the main component of the s-process, whose astrophysical site has been envisaged in the convective He shell of Thermally Pulsing Asymptotic Giant Branch (TP-AGB) stars of low mass (1-3 $M_{\odot}$; see Käppeler et al. 1990). The main neutron source is the ${ }^{13} \mathrm{C}(\alpha, n){ }^{16} \mathrm{O}$ reaction, operating at the thermal energy of $k T=12 \mathrm{keV}$. We have calculated neutron captures in such environment with an updated nuclear physics, adopting the neutron capture cross sections of Beer, Voß, \& Winters (1992) together with their temperature-dependence. Stellar models producing a mean neutron exposure of $\tau_{0} \simeq 0.30 \mathrm{mb}^{-1}$ are able to reproduce the solar distribution of the $s$ abundances satisfactorly, but the $\mathrm{Ba}$ isotopes show some overproduction. Such a strong indication suggests a revision of the $\mathrm{Ba}$ cross sections (see Gallino, Raiteri, \& Busso 1992). Once that a suitable choice of $\sigma_{n, \gamma}(\mathrm{Ba})$ is made, it is found that a $r$-contribution to solar $\mathrm{Ba}$ of the order of $10 \%$ can be expected.

We also investigated the production of $\mathrm{Ba}$ in stellar models of different neutron exposures, corresponding to stars of various metallicities. A constant amount of the ${ }^{13} \mathrm{C}$ neutron source was assumed. The results of our calculations are shown in Table I: the overabundances of the $\mathrm{Ba}$ isotopes are given with respect to the initial (solar-scaled) ones. From these numbers the Ba yields from TP-AGB stars can be derived; by inserting them into a detailed model for the chemical evolution of the Galaxy (Matteucci \& François 1989), we can predict the behaviour of barium as a function of metallicity. The results, that must be compared with the observation of $[\mathrm{Ba} / \mathrm{Fe}]$ vs. $[\mathrm{Fe} / \mathrm{H}]$, will be presented in a forthcoming paper.

\section{TABLE I}

\begin{tabular}{lcccccccc}
\hline$[\mathrm{Fe} / \mathrm{H}]$ & -1.3 & -0.82 & -0.51 & -0.35 & -0.22 & -0.12 & -0.05 & 0.0 \\
\hline${ }^{134} \mathrm{Ba}$ & $6.75 \mathrm{e} 3$ & $3.98 \mathrm{e} 3$ & $1.72 \mathrm{e} 3$ & $9.15 \mathrm{e} 2$ & $4.88 \mathrm{e} 2$ & $2.73 \mathrm{e} 2$ & $1.60 \mathrm{e} 2$ & $1.14 \mathrm{e} 2$ \\
${ }^{135} \mathrm{Ba}$ & $1.73 \mathrm{e} 3$ & $9.91 \mathrm{e} 2$ & $4.04 \mathrm{e} 2$ & $2.07 \mathrm{e} 2$ & $1.07 \mathrm{e} 2$ & $5.86 \mathrm{e} 1$ & $3.35 \mathrm{e} 1$ & $2.36 \mathrm{e} 1$ \\
${ }^{136} \mathrm{Ba}$ & $7.29 \mathrm{e} 3$ & $4.19 \mathrm{e} 3$ & $1.74 \mathrm{e} 3$ & $8.96 \mathrm{e} 2$ & $4.65 \mathrm{e} 2$ & $2.54 \mathrm{e} 2$ & $1.45 \mathrm{e} 2$ & $1.02 \mathrm{e} 2$ \\
${ }^{137} \mathrm{Ba}$ & $6.24 \mathrm{e} 3$ & $2.51 \mathrm{e} 3$ & $9.28 \mathrm{e} 2$ & $4.59 \mathrm{e} 2$ & $2.31 \mathrm{e} 2$ & $1.24 \mathrm{e} 2$ & $7.01 \mathrm{e} 1$ & $4.91 \mathrm{e} 1$ \\
${ }^{138} \mathrm{Ba}$ & $1.25 \mathrm{e} 4$ & $5.30 \mathrm{e} 3$ & $1.80 \mathrm{e} 3$ & $8.21 \mathrm{e} 2$ & $3.83 \mathrm{e} 2$ & $1.92 \mathrm{e} 2$ & $1.02 \mathrm{e} 2$ & $6.90 \mathrm{e} 1$ \\
\hline$\tau_{0}\left(\mathrm{mb}^{-1}\right)$ & 1.19 & 0.47 & 0.29 & 0.23 & 0.19 & 0.17 & 0.15 & 0.14 \\
\hline
\end{tabular}

\section{References}

Beer, H., Voß, F., \& Winters, R.R. 1992, ApJS, 80, 403

Gallino, R., Raiteri, C.M., \& Busso, M. 1992, ApJ, (submitted)

Käppeler, F., Gallino, R., Busso, M., Picchio, G., \& Raiteri, C.M. 1990, ApJ, 354, 630

Matteucci, F. \& François, P. 1989, MNRAS, 239, 885 